\title{
Améliorations juridiques en vue de favoriser la mise en place d'ouvrages de franchissement des obstacles par les poissons
}

\author{
Legal improvements aimed at facilitating \\ the installations of overpasses for fish
}

\author{
P. Denozière \\ Ingénieur Général honoraire \\ du Génie Rural, des Eaux et des Forêts
}

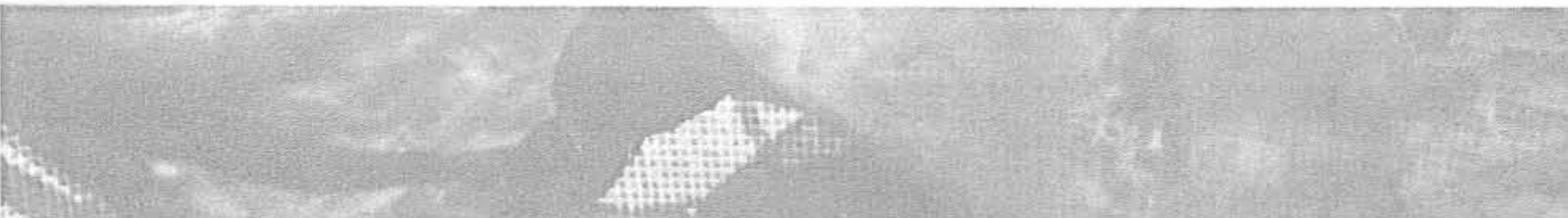

Pour le moment ces améliorations sont essentiellement contenues dans la nouvelle loi sur la pêche et principalement dans l'article 411 nouveau du Code Rural; cet article requiert des textes d'application qui ne sont pas encore connus.

Néanmoins les importantes mesures nouvelles de l'article 411 doivent permettrent que de nombreuses améliorations souhaitables, entre autres la restauration du franchissement dans les ouvrages antérieurs mal entretenus ou même sans alimentation en eau, soient obtenues; toutefois les modalités en sont incertaines faute de la connaissance des textes d'applications, le seul recours possible pour obtenir des renseignements consistant en l'interprétation de la loi à la lumière de ses travaux préparatoires.

Parallèlement un allègement des procédures d'autorisation ou de concession des ouvrages en cause devrait être recherché. En outre, en dehors de la législation sur la pêche, la loi du 15 juillet 1980 modifiant celle du 16 octobre 1919 et ses textes d'application apportent des possibilités distinctes d'améliorations juridiques du franchissement des ouvrages qu'il faudra rechercher.
\end{abstract}

Currently, these improvements are essentially contained in the new fishing Law and mainly in article 411 of the new Rural Code. This article requires the enforcement of texts which are not yet known.

However, the important measures of article 411 ought to allow many desirable improvements to be made including the restoration of overpasses in previous works which were poorly maintained or even had no water supply. Nevertheless, the related ways and means are uncertain because the content of the texts enforced are not yet known, this being the only possible way to obtain information about the interpretation of the Law in the light of the preparatory works.

At the same time, a concession relating to the authorization procedures or concession of works in question ought to be sought. Moreover, apart from the legislation on fishing, the Act of 15 July 1980 modifying that of 16 October 1919 and its applicable legal texts provide distinct possibilities for legal improvements relating to the crossing of works to be obtained. 
Après les exposés techniques présentés à la session dont il ressort que sur le plan technique les multiples études entreprises ont abouti à des solutions satisfaisantes pour le franchissement des ouvrages en rivière par les migrateurs, le présent exposé doit porter sur des améliorations juridiques susceptibles de favoriser ce franchissement.

Ces améliorations doivent se traduire par des textes réglementant les dispositifs de franchissement. Certes il ne peut être envisagé de susciter au cours de la présente session des propositions de textes juridiques fondamentaux en la matière, qui seraient du domaine législatif, d'autant plus que cette novation figure précisément dans la nouvelle loi sur la pêche du 29 juin 1984, grâce au nouvel article 411 du Code rural qui fera l'objet de la première partie du présent exposé.

$\mathrm{Si}$ cet article les principes d'une nouvelle réglementation, le caractère très général de ces principes requiert des textes d'application pour qu'ils puissent être effectivement applicables.

En ce domaine l'arrêté du ministre chargé de la pêche prévu au deuxième alinéa de l'article 411 fixant la liste d'espèces migratrices par bassin est intervenu le 2 janvier 1986 (J.O. du 4 février 1986).

En revanche pour l'application des principes de la réglementation il n'est intervenu qu'une simple circulaire du Ministre de l'Environnement n ${ }^{\circ} 85 / 3250$ du 27 novembre 1985, non publiée à notre connaissance au Journal Officiel; si cette circulation lie correctement l'aménagement des passes à poissons aux règlements d'eau des ouvrages, certaines de ses dispositions du moins paraissent empiéter sur le domaine règlementaire par les contraintes imposées aux particuliers ou même en certains points excéder celles exprimées par le législateur.

Quoiqu'il en soit, dans la présente étude, il sera essentiellement fait appel aux travaux préparatoires de la loi, lorsqu'ils expriment clairement l'intention du législateur sans contredire le sens du texte promulgué.

La deuxième partie de l'exposé portera sur les autres textes législatifs en la matière, y compris leurs textes d'application qui sont intervenus dont on peut espérer qu'ils permettront de profiter, du moins partiellement, de la novation de l'article 411 du Code Rural.

\section{L'article 411 nouveau du Code rural}

Le texte de l'article 411 est le suivant :

«Dans les cours d'eau ou parties de cours d'eau et canaux dont la liste est fixée par décret, après avis des Conseils généraux rendus dans un délai de six mois, tout ouvrage doit comporter des dispositifs assurant la circulation des poissons migrateurs. L'exploitant de l'ouvrage est tenu d'assurer le fonctionnement et l'entretien de ces dispositifs ".

"Les ouvrages existants doivent être mis en conformité, sans indemnité, avec les dispositions du présent article dans un délai de cinq ans à compter de la publication d'une liste d'espèces migratrices par bassin ou sous-bassin fixée par le ministre chargé de la pêche en eau douce et, le cas échéant, par le ministre chargé de la mer. "
A l'article précédent il y a lieu de joindre figurant dans la loi sur la pêche :

- l'article 412 du Code rural qui institue des amendes correctionnelles, assorties d'astreintes en cas de défaut d'exécution des mesures fixées par le juge, assurant ainsi l'efficacité des mesures prescrites pour l'application de l'article 411,

- l'article 12 de la loi sur la pêche d'après lequel le classement des cours d'eau intervenu en application de l'article $428-2^{\circ}$ ancien du Code rural, vaut classement au titre de l'article 411 nouveau.

\subsection{Quel est le champ d'application de l'article 411 ?}

Les dispositions de l'article 411 sont susceptibles d'être appliquées à tous cours d'eau ou canaux visés à l'article 402 du Code rural quel que soit leur statut juridique et donc tant pour les ouvrages situés sur les eaux domaniales que sur les eaux non domaniales.

\subsection{Améliorations apportées par l'article 411 à la réalisation du franchissement des migrateurs.}

1.2.1. En premier lieu la procédure de classement des cours d'eau sur lesquels s'applique l'article 411 est simplifiée.

Au lieu d'un classement, après enquête, par décret en Conseil d'Etat, comme il était prévu dans l'ancien article $428-2^{\circ}$, ce classement a lieu désormais par décret simple, sans mention d'enquête préalable.

\subsubsection{L'obligation de résultat}

L'ancienne rédaction disposait que sur les cours d'eau classés «il peut être établi... un passage appelé échelle, destiné à assurer la libre circulation du poisson " .

La nouvelle rédaction (« tout ouvrage doit comporter des dispositifs assurant la circulation des poissons migrateurs ") exprime une obligation de résultat, ce que confirment les travaux préparatoires (1).

Il en résulte en principe pour le propriétaire de l'ouvrage la liberté du choix des moyens pour parvenir au résultat. Les travaux préparatoires de loi le confirment par l'interprétation du mot " dispositifs" donné comme équivalent au choix de "dispositions" qui peuvent conduire le cas échéant au transport, extérieur aux ouvrages, des poissons par tous moyens (ascenseurs ou véhicules) (2).

En sens inverse des facilités données pour le franchissement par l'article 411 il faut noter la restriction de son texte aux poissons migrateurs par rapport au texte de l'article $428-2^{\circ}$, et la publication d'une liste d'espèce migratrices par bassin ou sous-bassin fixée par arrêté ministériel. Certes cette liste n'étant mentionnée que dans le $2^{\mathrm{e}}$ alinéa de l'article 411 peut ne paraître concerner que les ouvrages existants. Or non seulement cette solution est

(1) Cf. page 37 du rapport $n^{\circ} 1868$ de $M$. le député Colin du 10 décembre 1983.

(2) Assemblée nationale - Séance du 16 mai 1984. J.O. du 17 mai - p. 2405 , Déclarations du rapporteur et du Secrétaire d'Etat - Noter également avant le mot "circulation" la sup pression à cet effet du mot "libre" en commission mixte paritaire : Assemblée nationale $2^{\circ}$ séance du 5 juin 1984. p. 2931. 
illogique mais elle est contraire à l'intention du législateur, les documents des travaux préparatoires confirmant que la liste a pour but l'adaptation des dispositifs de franchissement aux diverses espèces migratrices, soit existantes, soit programmées en vue d'une restauration de la vie piscicole (3).

La liste des espèces a donc une portée générale, conditionne le classement des cours d'eau au titre de l'article 411 et s'applique tant aux ouvrages futurs qu'aux ouvrages existants : il convient donc, dans la lecture de l'article, de considérer les dispositions combinées des deux alinéas (4).

1.2.3. L'obligation des dispositifs de franchissement porte sur " tout ouvrage " et non plus, comme auparavant, seulement sur les «barrages".

1.2.4. Pour les ouvrages existants l'obligation de mise en conformité ne comporte pas d'indemnité d'après l'article 411. Cette exclusion d'indemnité, contrairement aux dispositions de l'article 429 ancien, peut-être cependant discutée en certains cas, ainsi qu'il sera exposé plus loin au paragraphe 3.4.

\subsection{Problèmes particuliers relatifs à la mise en auvre de l'article 411}

\subsubsection{Délais de mise en œuvre}

S'il résulte du texte que la mise en conformité des ouvrages existants doit être faite dans le délai de 5 ans à compter de la publication de l'arrêté ministériel relatif aux espèces migratrices, aucun délai n'est indiqué pour les nouveaux ouvrages : si donc pour ces derniers la loi peut paraître applicable dès son entrée en vigueur, malgré l'absence d'injonction spéciale du législateur sauf en ce qui concerne la liste des espèces migratrices, il peut apparaître contestable que la loi soit applicable avant l'intervention de certains textes réglementaires d'application lorsque les règles de sa mise en œuvre imposent aux particuliers des contraintes que la rédaction législative n'a pas explicitées.

\subsubsection{Fixation des débits consommés par les dispositifs de franchissement}

Ces débits, dont la fixation de l'ampleur est particulièrement importante pour les exploitants des ouvrages devront être fixés concrètement, que ce soit pour les dispositifs eux-mêmes ou pour les débits d'attrait vers ces dispositifs. Les débits peuvent se confondre avec le débit minimal réservé prescrit par l'article 410 mais il se peut qu'ils exigent en certains cas une majoration de ce dernier.

(3) Cf. Déclaration du rapporteur au Sénat - Séance du 19 avril 1964 - J.O. du 20 avril p. 430 - Déclaration du Secrétaire d'Etat à l'Assemblée nationale - 16 mai 1984 J.O. du 18 mai p. 2403 .

(4) La réponse du ministre chargé de l'environnement à la question écrite d'un parlementaire confirme expressément l'interprétation restrictive ci-dessus (Assemblée nationale J.O. du 3 novembre 1986, page 4064 . Question $\mathrm{n}^{\circ} 8496$ ).

(5) Cf. Rapport $n^{\circ} 1868$ pour l'Assemblée nationale - p. 16 - Débats en $2^{\mathrm{c}}$ lecture du Sénat (J.O. du 20 avril 1984 p. 43).

\subsubsection{Procédure de mise en œuvre des dispositifs}

$\mathrm{Si}$ une procédure spéciale est instituée par les textes d'application de l'article 411 pour la mise en œuvre des dispositifs, sauf dans le cas sûrement rare où ceux-ci seraient entièrement extérieurs aux ouvrages en rivière et n'entraineraient pas de consommation d'eau, cette procédure devrait être liée nécessairement à celle d'autorisation ou de concession de ces ouvrages, qu'il s'agisse d'une première autorisation ou concession ou, pour les ouvrages existants, d'une modification des autorisations ou des concessions, accordées les unes et les autres conformément aux règles légales et réglementaires en vigueur.

\subsubsection{Le problème de l'indemnisation pour les ouvrages existants.}

Comme il a été indiqué, l'article 411 exclut l'indemnisation pour les ouvrages existants. Cette exclusion a été motivée dans les travaux préparatoires par le Secrétaire d'Etat, d'une part par l'opportunité économique en raison des charges financières qui rendraient impossible la mise en conformité, d'autre part par référence à la règle qui interdirait l'indemnisation pour les mesures imposées au titre de l'environnement, application qui pourrait être contestée en l'espèce (5)

Quoiqu'il en soit, le problème juridique de l'indemnisation existe du moins pour des ouvrages situés sur les cours d'eau non domaniaux, du fait de la double formulation qui existe dans la loi sur la pêche en raison de son article 9 complétant l'article 109 du Code rural par un paragraphe $5^{\circ}$ par lequel «les autorisations ou permissions... peuvent être révoquées ou modifiées sans indemnité de la part de l'Etat exerçant ses pouvoirs de police dans les cas suivants :... $5^{\circ}$. Pour des raisons de protection de l'environnement et notamment lorsque ces autorisations soumettent les milieux naturels aquatiques à des conditions hydrauliques critiques, non compatibles avec leur préservation, selon les modalités fixées par décret en Conseil d'Etat ".

Or, ainsi qu'il a été indiqué la mise en conformité suppose la modification de l'autorisation qui rentre dans les cas limitativement énumérés par l'article 109 pour qu'il $\mathrm{y}$ ait dispense d'indemnité.

Il a y a donc deux formulations sur l'absence d'indemnisation, problème de conflit de lois dont la solution, qui incombe au juge, n'est pas facile.

\subsubsection{Problèmes divers}

Le problème a été posé de la restauration des ouvrages de franchissement antérieurs mal entretenus ou même sans alimentation en eau : ils rentreront dans le cas de la mise en conformité du deuxième alinéa de l'article 411. Par ailleurs il a été signalé, à juste titre, que de longs délais seraient requis en raison de la lenteur des procédures prescrivant les travaux.

Il a été noté en effet que les autorisations, qu'il s'agisse de nouveaux ouvrages ou d'ouvrages existants, doivent être accordés conformément aux textes en vigueur qui exigent effectivement des délais importants pour les autorisations sur les cours d'eau non domaniaux. Ces délais sont dûs en grande partie à l'archaïsme de ces textes: modèle d'autorisation du 26 décembre 1884 et procédure instituée par le décret du $1^{\text {er }}$ août 1905. 
Des projets ont été étudiés depuis de nombreuses années pour les moderniser et les simplifier mais n'ont pas encore pu aboutir : il serait souhaitable que la nécessité de la mise en œuvre de l'article 411 en facilite la parution.

\section{Autres textes relatifs au franchissement des poissons migrateurs}

\subsection{Cours d'eau réservés}

Le $5^{e}$ alinéa de l'article 2 de la loi du 16 octobre 1919 relative à l'utilisation de l'énergie hydraulique, à la suite de la modification apportée par l'article 25 de la loi $\mathrm{n}^{\circ} 80531$ du 15 juillet 1980, avait réservé certains cours d'eau, par ailleurs classés en vertu de l'ancien article 428- $2^{\circ}$ du Code rural, dont la liste serait établie par décret en Conseil d'Etat, sur lesquels aucune autorisation ou concession ne serait accordée pour des entreprises hydrauliques nouvelles.

L'Assemblée nationale, en sa $2^{\epsilon}$ lecture de la loi sur la pêche, a décidé sur proposition de son rapporteur (6) de supprimer, par son article 8-III, dans l'article 2 de la loi du 16 octobre 1919 les mots "classés en application de l'article $428-2^{\circ}$ du Code rural $»$.

Il en résulte que ces cours d'eau, sur lesquels les barrages pourraient être soumis à l'obligation d'installer les dispositifs de franchissement, sont désormais soumis, par un classement distinct par décret en Conseil d'Etat, à un régime spécial, plus strict, d'interdiction de nouvelles installations hydrauliques. Cependant si lesdits cours d'eau sont également classés au titre de l'article 411 nouveau, les ouvrages existants devront être mis en conformité en vertu de son $2^{c}$ alinéa.

\subsection{Autres cours d'eau non classés au titre de l'article 411}

Le cas peut se présenter, du moins temporairement, de problèmes de franchissement de migrateurs sur d'autres cours d'eau que ceux classés au titre de l'article 411 .

Les textes antérieurs concernant les autorisations et concessions d'ouvrages avaient déjà prévu ce problème du franchissement des poissons :

- pour les autorisations accordées conformément au modèle du 26 décembre 1884, qui concernent la très grande majorité des petites usines hydrauliques autorisées avant la loi de 1919 et maintenues sous leur régime antérieur en vertu de son article 19 , l'article 10 du modèle restreint cependant l'obligation du franchissement aux cas prévus par la loi du 31 mai 1865 à laquelle a succédé l'article $428-2^{\circ}$ du Code rural. La situation est la même pour les usines autorisées entre la loi de 1919 (article 8 du modèle de règlement d'eau du 8 janvier 1921) et les décrets 81.375 et 81.376 du 15 avril 1981 ;

- pour les usines autorisées après les décrets de 1981 la situation, non exempte d'ambiguïté, est différente.

Si la circulaire du 15 avril 1981 commentant les décrets du même jour " rappelle " (paragraphe 1.1) qu'au titre de l'article $428.2^{\circ}$ du Code rural les ouvrages doivent comporter des installations de franchissement des migrateurs, cas qui rentre maintenant dans celui de l'article 411 , le modèle de règlement d'eau approuvé par le décret 81.376 (article $7 b)$ prescrit que «le permissionnaire établira et entretiendra des dispositifs destinés à assurer la libre circulation du poisson " sans comporter la restriction du classement de l'article $428-2^{\circ}$. Ainsi toutes les nouvelles autorisations reprendraient en quelque sorte les dispositions spéciales en vigueur sous le régime de l'ancien article $428-2^{\circ}$. On peut d'ailleurs espérer que, l'exemple aidant, les acquis des nouveaux dispositifs prescrits par l'article 411 serviront de modèle et pourront ainsi, du moins partiellement, s'étendre aux autres autorisations.

Il est même prévu dans l'article $7 \mathrm{~b}$ du modèle de réglement d'eau que des indemnités annuelles d'alevinage pourront être imposées aux permissionnaires, sauf si les dispositifs de franchissement sont suffisants et en tout état de cause si la puissance de la chute est inférieure à $500 \mathrm{~kW}$.

- Pour les usines concédées il n'y a pas eu de modifications des textes en vigueur, à savoir l'article 7 du cahier des charges type de concession.

(6) Rapport n' 2102 de M.G. Colin p. 23.
M. Le Président. - Nous allons passer au deuxième exposé de synthèse, juridique cette fois.

Nous avons fait appel à une personnalité que vous connaissez tous car nous avons tous été ou élèves, ou auditeurs, de $\mathrm{M}$. DENOZIÈRE qui est Ingénieur général honoraire du GREF et Président de la Commission centrale de législation des eaux, placée au Ministère de l'Agriculture. C'est dire que ses attributions et ses compétences font de lui la personne adéquate pour faire cet exposé de synthèse sur la législation. Je lui donne la parole.

Exposé de M. Denozière 
M. Le Président. - Je comprend la difficulté de cet exposé : c'est qu'il faut être abonné au Journal Officiel... L'actualité a été riche depuis le début de l'année... L'exposé invoque même le Journal Officiel du 14 mars, qui apporte des modifications importantes.

M. ROGUET, - Jaimerais savoir ce que vous pensez de l'échéance de 1994 ?...

Avant 1919 il y a eu une législation qui a prorogé les autorisations en cours jusqu'en 1994. Cela correspond très souvent à des ouvrages très dangereux parce que sans débit réservé et sans échelles.

Comment voyez-vous les moyens juridiques à tirer de l'article 16 de la loi de 1919.

M. DENOZIÉRE. - Larticle 16 de la loi du 16 octobre 1919 fixe pour l'avenir à 75 ans la durée maximum des nouvelles autorisations d'usines hydrauliques. L'échéance de 1994 est surtout importante en vertu de l'article 18 de ladite loi qui maintient pendant 75 ans les usines alors autorisées sous leur régime antérieur si leur puissance est d'au moins $150 \mathrm{~kW}$ les autres usines (celles autorisées de moins de $159 \mathrm{~kW}$ ou celles fondées en titre) demeurent sous leur régime antérieur sans limitation de durée.

Quoi qu'il en soit toutes ces usines demeurent soumises à la législation sur les eaux et sur les cours d'eau non domaniaux leurs autorisations peuvent donc être modifiées, ou' même révoquées (article 109 du Code Rural, notamment son nouveau paragraphe 5); elles peuvent donc être modifiées en vertu de l'article 411 du Code Rural, lorsque le classement requis pour décret du cours d'eau est intervenu.

M. PICARD. - Dans les autres domaines du Droit français, lorsque quelqu'un ne respecte pas la réglementation, non seulement il est pénalisé pour ne pas avoir respecté cette réglementation, mais en outre on peut se substituer à lui pour faire les travaux à ses frais. Il ne semble pas que ce soit la solution adoptée dans le cas que vous évoquez, puisque même s'agissant des cours d'eau domaniaux il y a une obligation d'indemnisation, si l'on fait perdre de l'argent à quelqu'un...

M. DENOZIÉRE. - Vous posez la question de l'exécution d'office des travaux de passes à poissons. Dans le cas d'autorisations d'ouvrages hydrauliques privés, accordées sur des cours d'eau non domaniaux non classés au titre de l'article 411 suivant le modèle du 26 décembre 1884 toujours en vigueur, qu'il s'agisse d'usines maintenues sous leur régime antérieur ou d'ouvrages d'autre nature, l'exécution d'office qui n'est prévue ni dans la loi (le Code Rural), ni dans un décret, ni même dans le modèle d'autorisation, n'est pratiquement pas possible.

La situation est plus favorable dans le cas des cours d'eau classés de l'article 411 , l'article 412 prévoyant l'exécution d'office sur décision du juge pénal; pour les nouvelles autorisations d'usine en application du décret du 15 avril 1981 l'exécution d'office est prévue par l'article 20 de ce décret, mais assortie de certaines conditions. Quoiqu'il en soit, même avec des conditions juridiques favorables, la réalisation de l'exécution d'office n'est pas une opération facile.

M. PICARD. - Est-ce que l'exécution d'office pourrait être applicable ?

M. DENOZIÉRE. - La déchéance ainsi que la mise en chômage des installations figurent dans le modèle d'autorisation de 1884 qui vient d'être évoqué et concernent de très nombreux ouvrages, sanctions prévues pour le cas de non respect des clauses des autorisations, dont la menace pourrait avoir un effet dissuasif, mais elles ne sont plus pratiquement appliquées. Encore faudrait-il d'ailleurs que l'autorisation prévoie l'installation d'une échelle à poissons, mais d'après le modèle d'autorisation cette obligation ne peut être prescrite que lorsque la législation spéciale sur la pêche l'exige, c'est-à-dire, maintenant, dans le cas des cours d'eau classés de l'article 411 .

Pour les nouvelles autorisations d'usines soumises au décret du 15 avril 1981, le terme de déchéance est remplacé par celui de retrait d'office de l'autorisation, ce qui revient pratiquement au même. Le modèle d'autorisation prévoit également la sanction de la mise en chômage : en revanche l'obligation d'installation de dispositifs de franchissement des poissons est inscrite au modèle sans la restriction d'être limitée à la soumission du cours d'eau à un régime spécial de la législation sur la pêche.

En définitive les textes en vigueur prévoient bien les sanctions administratives indiquées mais sans qu'il soit sûr que l'administration soit disposée à y recourir.

M. ROGUET. - Je voudrais signaler encore un décret récent... it date du 7 février 1986... C'est à propos de l'application de larticle 8 bis de la loi du 8 avril 1946.

La loi «Pêche" a prévu qu'E.D.F. ne pouvait acheter l'énergie que si les installations étaient régulièrement autorisées ou concédées.

Et bien ! cette mise en œuvre par un décret qui vient de sortir a déjà produit des effets : on a vu beaucoup de propriétaires déposer des dossiers de régulation.

Maintenant je voudrais poser une question à E.D.F. :

Dans la mesure oủ une micro-centrale vend du courant à E.D.F., il y a un contrat liant le producteur avec E.D.F.; et dans ce contrat est indiquée la puissance de l'installation.

Or nous avons pu constater à diverses reprises, pour des dossiers de mise en conformité, que la puissance qui figure sur le contrat d'achat passé avec E.D.F. était de beaucoup supérieure à celle autorisée par l'Administration. Par exemple, certains industriels ont demandé à obtenir une autorisation de $500 \mathrm{~kW}$ pour des questions de procédure, mais ils en fabriquent $1500 \ldots$ et E.D.F. est au courant puisque cela figure dans son contrat d'achat.

M. WEISROCK. - Ce n'est pas Electricité de France qui a la charge de faire appliquer la réglementation.

L'Etablissement n'a pas le droit de se substituer à l'Administration.

Le point est donc clair :

- d'un côté, E.D.F. est obligé, par la loi, d'acheter l'énergie des producteurs autonomes;

- d'un autre, E.D.F est tenu d'interrompre le contrat d'achat d'énergie dès que l'Administration le lui demande suite à constat par cette dernière d'irrégularités dans le fonctionnement de la centrale.

M. Le Président. - S'il y a d'autres questions, elles pourront être posées à la table ronde.

Je voudrais maintenant remercier M. I'Ingénieur général DENOZIĖRE pour son exposé.

Vous avez senti avec quelle autorité il nous parlait des textes, et même du contexte dans lequel ils ont été étudiés au Parlement et dans les Ministères.

M. l'Ingénieur Général vient de me faire savoir qu'il était à votre disposition, si vous souhaitiez le consulter par écrit ou par téléphone sur des problèmes précis... Vous pouvez le joindre : 19 avenue du Maine, Paris $\left(15^{\circ}\right)$... Il est à votre disposition.

Malheureusement nous n'avons pas le temps de faire de pause; nous enchaînons immédiatement sur le film :

Présentation du film : Le saumon sur l'axe Loire/Allier

M. Le Président. - Je crois que vos applaudissements prouvent l'intérêt que vous avez trouvé à ce film à la fois technique, esthétique, et parfois même poétique. Il faut remercier M. CUINAT et son équipe pour sa réalisation.

$M$. Cuinat. - Je précise que ce film résulte d'une entente : il a été commandité par la Fédération de Pêche et le Ministère de l'Environnement. Nous aurions, nous, voulu évoquer des situations beaucoup plus futuristes que nous espérons connaître par la suite... Par exemple nous ne sommes pas satisfaits du coup de gaffe qu'on voit à la fin; dans l'avenir on ne fera plus saigner les saumons, on les sortira de l'eau de façon plus normale. 